\title{
Endovascular stent-graft repair for thoracoabdominal aneurysm after reconstruction of the superior mesenteric and celiac arteries
}

Yoshifumi Iguro, MD, Goichi Yotsumoto, MD, Naoki Ishizaki, MD, Kenichi Arata, MD, and Ryuzo Sakata, MD, Kagoshima, Japan

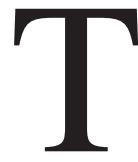

horacoabdominal aortic aneurysm (TAAA) is not considered on indication for endovascular stent-graft repair because of the need to revascularize the visceral vessels. This article details for the first time a case of TAAA repair in which an endovascular stent graft was placed after reconstruction of the visceral vessels.

\section{Clinical Summary}

A 63-year-old woman was referred to our hospital for the treatment of a TAAA. The patient had tuberculosis and pleuritis at 16 and 21 years of age, respectively. Computed tomography and abdominal echocardiography revealed a porcelain descending aorta and a large saccular calcified aneurysm with mural thrombus in the thoracoabdominal aorta. The maximum aneurysmal diameter was $60 \mathrm{~mm}$. Aortography showed a Crawford extent III TAAA involving the celiac artery and the superior mesenteric artery (SMA; Figure 1).

We assumed that pleural adhesion and severe calcification would make formation of the proximal and visceral vessel anastomosis difficult. We planned endovascular stent-graft repair to follow the extra-anatomic reconstruction of the visceral vessels by using the right iliac artery system as a pedicled graft. A midline celiotomy was made, and the SMA, hepatic artery, celiac axis, and bilateral iliac artery were dissected out in preparation for the bypass. The huge TAAA was palpated at just below the diaphragm, and a small $(25 \mathrm{~mm})$ aneurysm was recognized at the midportion of the infrarenal abdominal aorta. Neither the bilateral common nor the external iliac artery had any calcification on palpation, which had adequate length and quality for grafting to the SMA. After systemic heparinization, the right iliac artery system was completely dissected free from the surrounding tissues from the aortic bifurcation to the end of the external iliac artery, which was transected just above the inguinal ligament and brought up through the Trietz ligament to be used for a pedicled graft to the SMA. After the end-to-side anastomosis to the SMA was per-

\footnotetext{
From the Second Department of Surgery, Kagoshima University Faculty of Medicine, Kagoshima, Japan.

Received for publication July 10, 2002; accepted for publication Aug 20, 2002 .

Address for reprints: Yoshifumi Iguro, MD, The Second Department of Surgery, Kagoshima University, Faculty of Medicine, 8-350-1, Sakuragaoka Kagoshima City, Kagoshima Prefecture, 890-8520 Japan (E-mail: iguro@med6.kufm.kagoshimau.ac.jp).

J Thorac Cardiovasc Surg 2003;125:956-8

Copyright (C) 2003 by The American Association for Thoracic Surgery $0022-5223 / 2003 \$ 30.00+0$

doi: $10.1067 / \mathrm{mtc} .2003 .152$
}
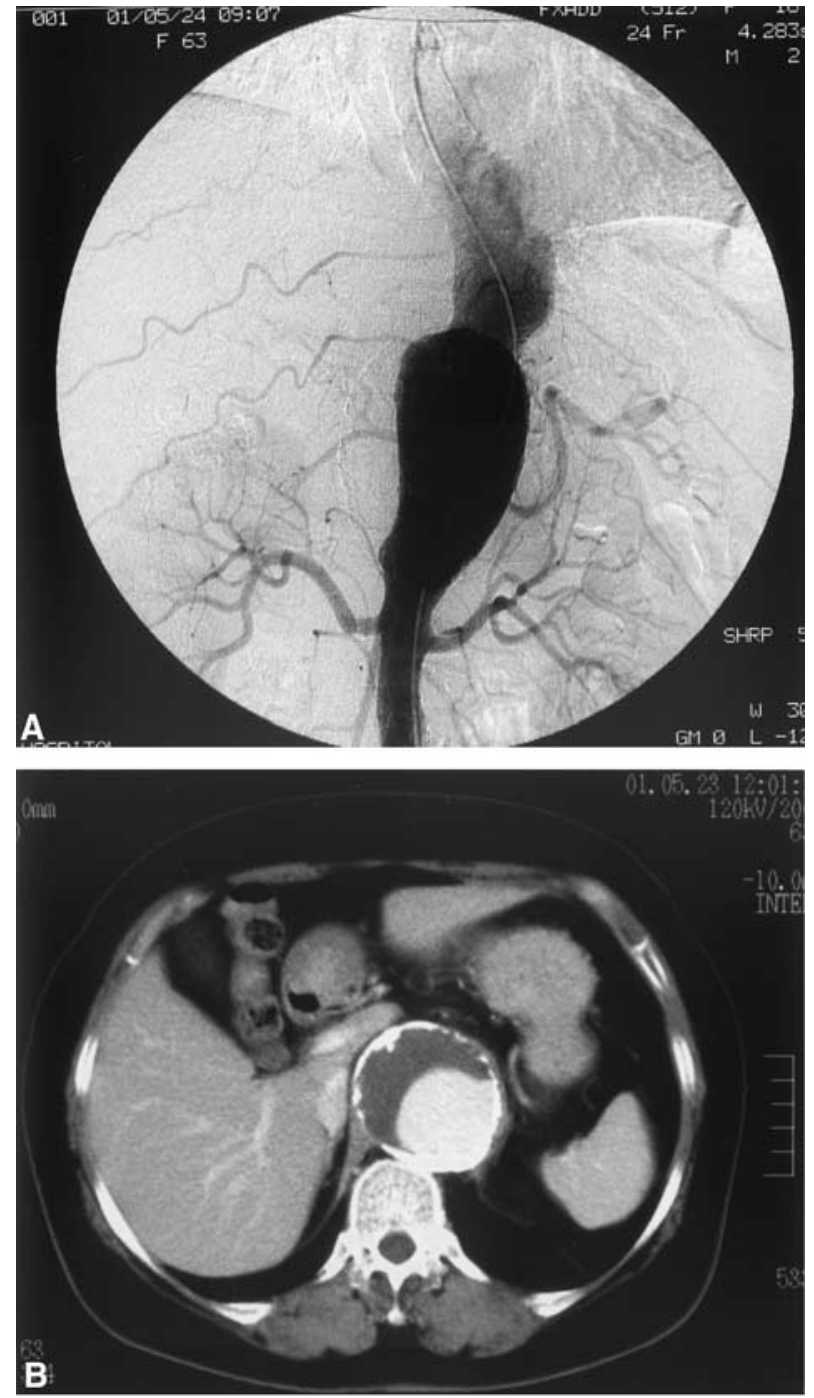

Figure 1. Preoperative aortogram and computed tomogram. A, Preoperative aortogram showing a Crawford grade 3 saccular TAAA arising from the supraphrenic descending aorta and extending to the suprarenal aorta. The aneurysm involved the celiac artery and the SMA. B, Enhanced computed tomogram showing a large saccular calcified aneurysm, with mural thrombus in the thoracoabdominal aorta. The maximum aneurysmal diameter was $60 \mathrm{~mm}$.

formed, the root of the SMA was ligated. The left greater saphenous vein was also harvested simultaneously for the bypass between the reverse pulled-up iliac artery and the common hepatic 

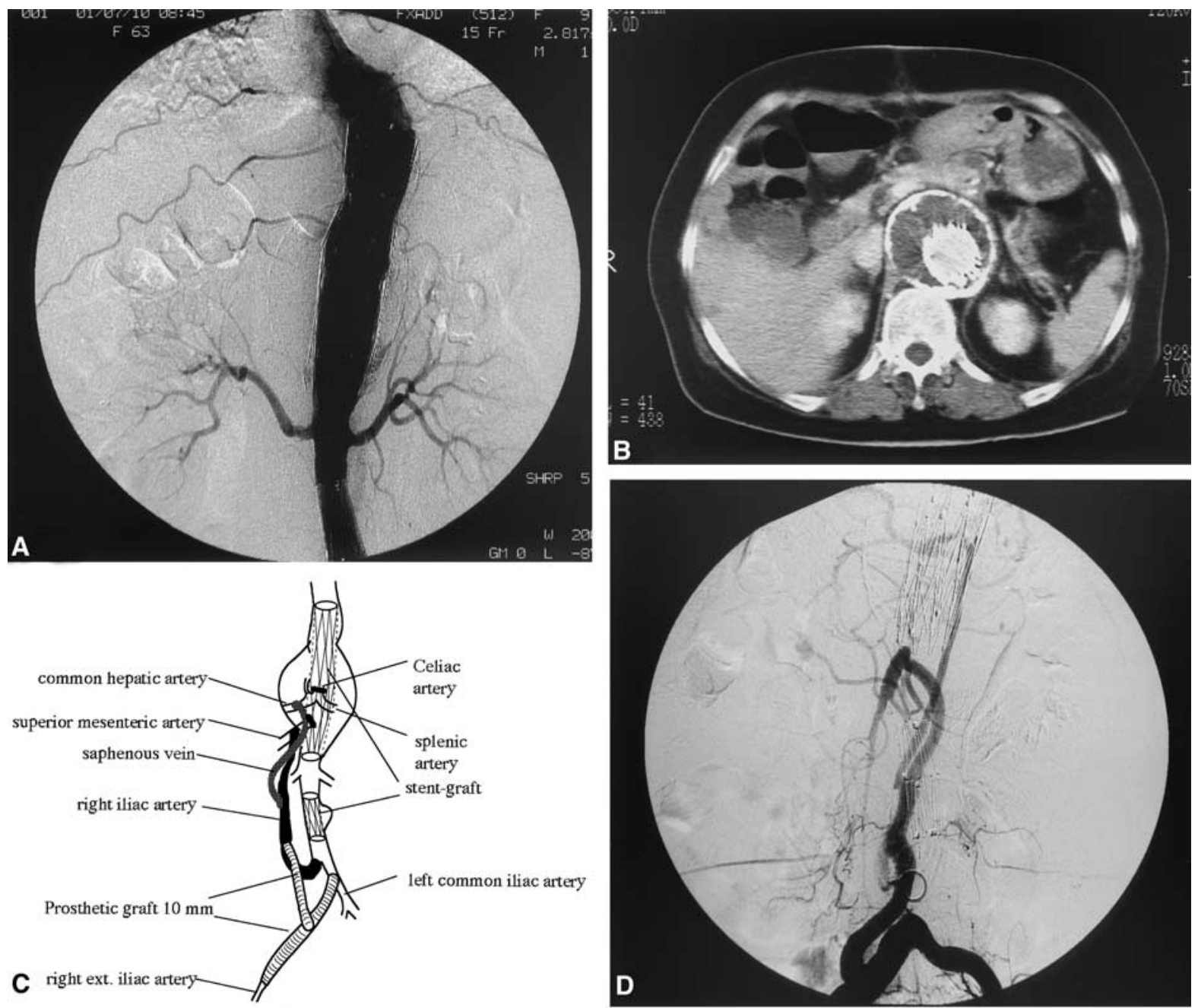

Figure 2. Postoperative aortogram and computed tomogram. A, Postoperative aortgraphy showing the disappearance of the saccular aneurysm and the normal flow of the bilateral renal artery. B, Computed tomogram showing complete clot formation of the aneurysm and no leakage. C, Illustration of the bypass and position of the stent graft. D, Postoperative selective angiography revealing the normal flow of the celiac artery and the SMA through the bypass.

artery. The celiac axis was ligated after the visceral vessel reconstruction was completed, and endoluminal stent-graft placement was done through a 10-mm-diameter prosthetic woven graft (Meadox Medicals, Inc, Oakland, NJ) anastomosed to the left common iliac artery. A $22 \mathrm{~F}$ sheath was inserted through this graft, and the stent graft was deployed into the TAAA. The stent graft was constructed from a self-expanding 40-mm-diameter Gianturco stainless-steel Z stent (Cook Group, Inc, Bloomington, Ind) and a thin-walled 32-mm-diameter woven Dacron vascular graft (Ubekosan, Inc). The stent graft was $100 \mathrm{~mm}$ in length. An additional stent graft $50 \mathrm{~mm}$ in length was deployed at the distal site because of minor leakage, resulting in complete exclusion of the aneurysmal lesion. The small aneurysm in the midportion of the infrarenal aorta was treated with another 28-mm-diameter stent graft deployed through the same $10-\mathrm{mm}$ prosthetic graft. Finally, this 10-mm prosthetic graft was anastomosed to the end of the right external iliac artery for reperfusion of the right lower extremity. A final intraoperative aortogram revealed complete exclusion of the 2 aneurysms and adequate blood flow to the visceral vessels.

During the next 24 hours, the patient had progressive liver dysfunction, and the transaminase level in the liver increased to 4000 units. Angiography revealed a stenosed kinking of the pedicled right iliac artery graft at its takeoff point, which was corrected by wrapping with a prosthetic graft to prevent further kinking. For insurance, we anastomosed a 10-mm prosthetic graft (Meadox Medicals, Inc) between the left common iliac artery bypass graft and the right common iliac artery.

Thereafter, the patient's liver function promptly returned to normal ranges, with restoration of the good general conditions. Three weeks after the stent-graft repair, complete exclusion of the aneurysms and patency of the bypass grafts were verified by means of aortography and computed tomography (Figure 2). She is doing 
well at 1-year follow-up with no evidence of visceral ischemia and continued exclusion of the aneurysms.

\section{Comment}

Conventional TAAA repair is a technically challenging operation with a significant morbidity rate. ${ }^{1}$ Repair of such complex aneurysms requires a difficult and extensive exposure and supraphrenic aortic crossclamping, as well as revascularization of visceral vessels and intercostal arteries at times. Endoluminal placement of a stent graft is advanced as a new and less-invasive alternative technique to conventional repair of the aortic aneurysm. ${ }^{2}$ However, this new technique is rarely applied to the patient with TAAA because of the need to reconstruct the visceral and intercostal arteries. In this case, considering the presence of severe pleural adhesions and calcified aneurysm involving visceral vessels, we used the new technique for stent grafting with simultaneous reconstruction of the visceral branches. Of course, our method leaves much room for improvement. Long-term careful follow-up is important because the common iliac artery, which was used as a bypass graft to the SMA, has been reported to have a tendency toward sclerosis.

In conclusion, our patient's TAAA was excluded successfully by means of the less-invasive implantation of a stent graft without extracorporeal circulation and thoracotomy. Our experience suggests that this approach has a possibility to expand the indications for endovascular repair of TAAA.

\section{References}

1. Derrow AE, Seeqer JM, Dame DA, Carter RL, Ozaki CK, Flynn TC. The outcome in the United States after thoracoabdominal aortic aneurysm repair, renal artery bypass, and mesenteric revascularization. $J$ Vasc Surg. 2001;34:54-61.

2. Dake MD, Miller DC, Mitchell RS, Semba CP, Moore KA, Sakai T. The first generation of endovascular stent-grafts for patients with aneurysms of the descending thoracic aorta. $J$ Thorac Cardiovasc Surg. 1998;116:689-704.

\title{
Abdominal aneurysm rupture and left main stenosis: Emergency one-stage repair with off-pump coronary surgery
}

\author{
Gerard Babatasi, MD, PhD, Massimo Massetti, MD, Jean-Michel Radoux, MD, and Andre Khayat, MD, Caen, France
}

oronary artery disease (CAD) and low ejection fraction are the most important risk factors for morbidity and mortality in patients undergoing abdominal aneurysm repair. Prior revascularization techniques have helped
to decrease the risk of cardiac-related adverse events. There is, however, a subgroup of patients having both severe CAD and an acutely expanding or ruptured aneurysm that represent a therapeutic challenge.

\section{Clinical Summary}

The patient was a 62-year-old man with increasing abdominal pain with posterior radiation. Computed tomographic scanning demonstrated an $8.9-\mathrm{cm}$ aneurysm and a perianeurysmal hematoma (Figure 1). The patient had a history of angina. Electrocardiography (ECG) demonstrated signs of myocardial ischemia. The hemoglobin level was $11.7 \mathrm{~g}$ initially and decreased to $9.4 \mathrm{~g}$ in 3 hours.

\footnotetext{
From the Cardiovascular Surgery Department, University Hospital CHU Caen, France.

Received for publication July 25, 2002; accepted for publication Aug 15 , 2002.

Address for reprints: Gérard Babatasi, MD, PhD, Cardiovascular Surgery Department, University Hospital, Avenue Côte de Nacre, CHU Caen, 14033 Caen cedex, France (E-mail: babatasi-g@chu-caen.fr).

J Thorac Cardiovasc Surg 2003;125:958-60

Copyright @ 2003 by The American Association for Thoracic Surgery $0022-5223 / 2003 \$ 30.00+0$

doi: $10.1067 / \mathrm{mtc} .2003 .150$
}

Coronary angiography (Figure 1) demonstrated left main coronary artery stenosis $(>75 \%)$. Ejection fraction was impaired $(45 \%)$. Although median sternotomy was first performed, the left internal thoracic artery (LITA) and radial artery were harvested simultaneously. No vein graft was available. Heparin (200 IU/kg) was administered. Heart exposure and stabilization was achieved by using the Cohn immobilizer (Genzyme Corporation, Cambridge, Mass). The LITA was first implanted to the left anterior descending artery. The circumflex artery was exposed in the atrioventricular groove, and the anastomosis was performed with the radial artery. The radial artery was implanted in the LITA ( $\mathrm{T}$ graft and end to side) to limit the potential risk of embolic catastrophe associated with crossclamping the severely diseased aorta. The chest was closed, and laparotomy was then performed. An extensive hematoma was detected on the retroperitoneal area. A standard graft inclusion technique with a collagen-coated Dacron bifurcated (18/9) graft (Hemashield, Meadox Medicals, Inc, Oakland, NJ) was performed. The length of the total procedure was 5 hours. Transfusions included 4 units of blood, $400 \mathrm{~mL}$ of fresh plasma, and 1 unit of platelets. ECG results returned to normal levels immediately after the cardiac procedure. The patient was extubated the day after the procedure. The patient was symptom free without any medication at 1 year of follow-up.

\section{Comments}

Surgical mortality from abdominal aortic aneurysm (AAA) repair has decreased over the past 20 years because of improvements in anesthetic technique, better intraoperative hemodynamic control caused by careful intensive monitoring, improved postoperative 